\title{
Roundabout Homolog 4
}

National Cancer Institute

\section{Source}

National Cancer Institute. Roundabout Homolog 4. NCI Thesaurus. Code C74446.

Roundabout homolog 4 (1007 aa, 107 kDa) is encoded by the human ROBO4 gene.

This protein plays a role in angiogenesis and blood vessel branching. 